\title{
Carnitine Palmitoyltransferase I Deficiency
}

National Cancer Institute

\section{Source}

National Cancer Institute. Carnitine Palmitoyltransferase I Deficiency. NCI Thesaurus.

Code C98871.

A rare autosomal recessive inherited disorder caused by mutations in the CPT1A gene. It is characterized by the presence of defective carnitine palmitoyltransferase $1 \mathrm{~A}$ which is involved in fatty acid oxidation. Signs and symptoms may be exacerbated during fasting and include hypoketotic hypoglycemia, increased levels of carnitine in the blood, hepatomegaly, seizures, and coma. 